\title{
Agreement analysis of LENSTAR with other techniques of biometry
}

S Jasvinder ${ }^{1}$, TF Khang ${ }^{2}$, KKS Sarinder², VP Loo ${ }^{1}$ and $V$ Subrayan

\begin{abstract}
Purpose To assess the agreement of the optical low-coherence reflectometry (OLCR) device LENSTAR LS900 with partial coherence interferometry (PCI) device IOLMaster and applanation and immersion ultrasound biometry.

Methods We conducted the study at the Ophthalmology Clinic, University of Malaya

Conclusions In phakic eyes of cataract patients, measurements of $A L$, average $K$, and IOL power calculated using the SRK/T formula from LENSTAR are biometrically equivalent to those from IOLMaster, but not with those from applanation and immersion ultrasound biometry. Eye (2011) 25, 717-724; doi:10.1038/eye.2011.28; published online 11 March 2011
\end{abstract} Medical Center, Malaysia. Phakic eyes of 76 consecutive cataract patients were measured using four different methods: IOLMaster, LENSTAR and A-scan applanation and immersion ultrasound biometry. We assessed the method agreement in the LENSTARIOLMaster, LENSTAR-applanation, and LENSTAR-immersion comparisons for axial length (AL) and intraocular lens (IOL) power using Bland-Altman plots. For average $K$, we compared LENSTAR with IOLMaster and the TOPCON KR-8100 autorefractor-keratometer. SRK/T formula was used to compute IOL power, with emmetropia as the target refractive outcome.

Results For all the variables studied, LENSTAR agreement with IOLMaster is strongest, followed by those with immersion and applanation. For the LENSTARIOLMaster comparison, the estimated proportion of differences falling within $0.33 \mathrm{~mm}$ from zero AL and within 1D from zero IOL power is $100 \%$. The estimated proportion of differences falling within $0.5 \mathrm{D}$ from zero average $\mathrm{K}$ is almost $100 \%$ in the LENSTAR-IOLMaster comparison but $88 \%$ in the LENSTAR-TOPCON comparison. The proportion of differences falling within $0.10 \mathrm{~mm}(\mathrm{AL})$ and within 1D (IOL power) in the LENSTAR-IOLMaster comparison has practically significant discrepancy with that of LENSTAR-applanation and LENSTARimmersion comparisons.
Keywords: LENSTAR; IOL Master; biometry

\section{Introduction}

With the introduction of new biometry techniques such as the partial coherence interferometry-based IOLMaster (version 5, Carl Zeiss, Jena, Germany) and the optical low coherence reflectometry-based LENSTAR LS900 (Haag-Streit, Bern, Switzerland), accurate, fast and easy measurement of ocular variables is now routine at many eye centres in industrialised nations. The optical biometry device IOLMaster, for example, can achieve axial length (AL) measurement precision within $20 \mu \mathrm{m},{ }^{1}$ compared to $100 \mu \mathrm{m}$ in ultrasound biometry. ${ }^{2}$ More importantly, the built-in computer algorithms in these devices allow intraocular lens (IOL) power calculation using a range of formulae, some of which require sophisticated optimisation procedures. ${ }^{3}$ This greatly extends the usefulness of these devices in both research and consultation work.

In contrast, ophthalmologists in developing countries continue to rely on applanation and immersion ultrasound biometry in clinical consultation. Skilled doctors or technicians can usually obtain accurate AL and IOL power measurements using these methods.

Nevertheless, variations in probe position, degree of corneal indentation, and patient movements during a biometry session can affect the final outcome. Furthermore, these methods
${ }^{1}$ Department of Ophthalmology, Faculty of Medicine, University of Malaya, Kuala Lumpur, Malaysia

${ }^{2}$ Institute of Biological Sciences, Faculty of Science, University of Malaya, Kuala Lumpur, Malaysia

Correspondence: $\checkmark$ Subrayan, Department of Ophthalmology, Faculty of Medicine, University of Malaya, 50603 Kuala

Lumpur, Malaysia Tel: + 6037949 2060; Fax: + 60379494635 E-mail: dvisva@hotmail.com

Received: 5 November 2010 Accepted in revised form: 7 January 2011 Published online: 11 March 2011 
require several steps before an IOL power measurement can be obtained, unlike the 'one-stop' new optical biometry devices. This provides opportunities for mistakes to creep in at each step (eg, when keying in the $\mathrm{K}$ readings into the A-scan machines). Patients may feel discomforted by the eye-probe contact in applanation biometry, which can also expose them to corneal abrasion or eye infection. ${ }^{4}$ Despite these shortcomings, ultrasound biometry may be the only means of obtaining suitable biometry in problematic cases: eyes with dense ocular media, ${ }^{5}$ low visual acuity, ${ }^{6}$ and fixation instability caused by macular degeneration. ${ }^{7}$ IOLMaster has been reported to be ineffective in $\sim 20 \%$ of cataract patients ${ }^{8}$ in the UK public hospitals, many of whom have posterior subcapsular cataracts.

When different ways of measuring the same variable are available, it is of interest to find out how well two different methods agree, as strong agreement implies that they can be used interchangeably. ${ }^{9}$ Assessing method agreement can also be useful for evaluating the relative importance of instrument variation as a source of error for IOL power. Several studies ${ }^{10-14}$ that look at LENSTAR-IOLMaster agreement in AL, anterior chamber depth (ACD), IOL power, K1, and K2 measurements of phakic eyes have been done. All suggest that measurements obtained from LENSTAR and IOLMaster strongly agree. These studies, however, do not tell us how good the agreement between LENSTAR and IOLMaster is relative to those of LENSTARapplanation and LENSTAR-immersion. In addition, the statistical arguments used to justify the conclusions in some cases are specious. Misconceptions about what constitutes method agreement remain prevalent: high correlation $^{15,16}$ and $R^{2}$ values from a linear regression analysis of the $x-y$ variables in the Bland-Altman $\operatorname{plot}^{17-19}$ are synonymous with strong agreement. The present study aims to address these gaps by comparing all three method agreement analyses for AL, average K, and IOL power using a coherent statistical approach.

\section{Materials and methods}

The present study was done at the Ophthalmology Clinic in the University of Malaya Medical Centre (UMMC), Kuala Lumpur, Malaysia. The first author, who is an experienced trainee ophthalmologist with 3 years of experience in biometry, performed all biometry work. To collect data, he approached patients in the clinic who had varying degrees of cataract severity in both eyes, and informed them about the purpose of the present study. Patients who gave informed consent and did not suffer from physically inconvenienced illnesses (ie, wheel-chair bound and limb injuries) were included in the study. The patients were first examined using IOLMaster (version 5), followed by LENSTAR, immersion and finally applanation. For an eye, if it was not possible to measure any of the ocular variables using IOLMaster because of very dense media along the visual axis, we excluded it from consideration. Measurements of $\mathrm{AL}, \mathrm{ACD}$ (not used), K1, and $\mathrm{K} 2$ were taken from both eyes; to calculate IOL power, we set emmetropia as the refractory target and used the SRK/T formula. ${ }^{20}$ For each eye (under miosis), five AL and three keratometry measurements were taken then averaged. IOL power calculation from A-scan applanation and immersion ultrasound biometry readings requires a separate assessment of keratometry values. For this, we used the TOPCON KR- 8100 autorefractor-keratometer (Topcon Europe Medical Bv, Capelle a/d ljssel, the Netherlands) to obtain K1 and K2 readings. The average time taken to obtain all readings for an eye in a patient was $\sim 2$ min for IOLMaster, three-and-a-half minutes for LENSTAR, and $4 \mathrm{~min}$ for applanation and immersion ultrasound biometry.

Data collection ceased when 76 consecutive patients who came for cataract evaluation had been sampled. We queried 104 patients, with a 73\% response rate. Reasons for declining to participate in the study include obesity, lack of time, and uneasiness with undergoing four biometry sessions in a sitting. The sampling period was from 12 May to 1 June 2010. The average age of the patients (27 females; 49 males) was 54 years, with SD 15 (range 18-86; see Supplementary Information). We certify that all applicable institutional and governmental regulations concerning the ethical use of human volunteers were followed during this research.

\section{Statistical analysis}

We compared how well measurements of AL and IOL power agreed in three pairwise comparisons: LENSTAR-IOLMaster, LENSTAR-applanation and LENSTAR-immersion. For the average $\mathrm{K}$ variable $((\mathrm{K} 1+\mathrm{K} 2) / 2)$, we made two comparisons: LENSTAR with IOLMaster and LENSTAR with the TOPCON autorefractor-keratometer. In determining the sample size for the present study (76), we used information from a preliminary assessment of measurement variation in the method comparison groups. First, we estimated the mean difference and SD of AL for all three comparison groups using 22 eyes from 13 patients in a similar study. We then computed the sample size required to achieve at least $80 \%$ power of detecting the mean difference with $\mathrm{SD}$ in each case (two-sided paired $t$-test, $5 \%$ significance level), and used the maximum of the three estimated sample sizes (74 pairs of eyes) as a guide. Pooling of left and right eyes for subsequent analysis seems reasonable, as most of the variation in the ocular variables considered is found within rather than between the left 
and right eyes (analysis of variance $P$-values: 0.8 (AL), 0.8 (IOL power), 0.9 (average K)). The total number of eyes in each comparison group varies from 142 to 147 because of three reasons: mistakes in data recording (misplaced printouts), single-eyed patients and dense cataract cases.

We used Bland-Altman plots to assess the level of agreement (width of the $95 \%$ limits of agreement, LoA). In order to relate observations from the Bland-Altman plots to a clinical context, we have to decide how narrow the LoA should be to conclude that two methods agree well. This is a clinical decision that is context-dependent and needs to be determined in advance of an analysis. ${ }^{9}$ Our approach is to evaluate the proportion of differences falling into particular ranges that show varying levels of clinical impact. Thus, we defined clinically acceptable ranges for IOL power (within 1D, between $1 \mathrm{D}$ and $2 \mathrm{D}$, greater than $2 \mathrm{D}$ ), $\mathrm{AL}$ (within $0.33 \mathrm{~mm}$, within $0.10 \mathrm{~mm}$ ), and average $\mathrm{K}$ (within $1 \mathrm{D}$, within $0.5 \mathrm{D}$ ) differences, and then estimated the proportion of differences falling within those ranges from the empirical distribution of differences. We chose these ranges based on the following reasoning. A difference of within $1 \mathrm{D}$ in IOL power corresponds to the case where patients can generally see well without corrective glasses; between $1 \mathrm{D}$ to $2 \mathrm{D}$, the patient would need corrective glasses of low power, but it is not hard to explain to the patient about the small error; above $2 \mathrm{D}$ represents the situation of the patient facing unacceptable postoperative high refractive error. Our choice of the $0.33 \mathrm{~mm}$ and $0.10 \mathrm{~mm}$ cut-off for $\mathrm{AL}$ is based on the fact that these differences translate to $\sim 1 \mathrm{D}$ and $0.3 \mathrm{D}$ difference in IOL power, respectively, under the SRK/T formula. Similarly, differences of $1 \mathrm{D}$ and $0.5 \mathrm{D}$ in average $\mathrm{K}$ translate to $\sim 1 \mathrm{D}$ and $0.5 \mathrm{D}$ difference in IOL power.

To judge whether the proportions of interest in two comparison groups are similar, we estimated their discrepancy (the larger value $\widehat{p_{1}}$ minus the smaller one $\left.\widehat{p_{2}}\right)$, and reported the SE, which is given by the formula ${ }^{9}$

$$
\mathrm{SE}\left(\widehat{p_{1}}-\widehat{p_{2}}\right)=\sqrt{\frac{\widehat{p_{1}}\left(1-\widehat{p_{1}}\right)}{n_{1}}+\frac{\widehat{p_{2}}\left(1-\widehat{p_{2}}\right)}{n_{2}}},
$$

where $n_{1}$ and $n_{2}$ are the sample sizes of the first and second comparison group, respectively. We used Fisher's exact test to detect association between comparison groups and several categories of clinically acceptable difference ranges for IOL power. All statistical work was done using $\mathrm{R}^{21}$ (version 2.10.1).

\section{Results}

The LENSTAR-IOLMaster comparison has the smallest magnitude of mean and SD of difference in AL (0. $01 \mathrm{~mm} \pm 0.03 \mathrm{~mm})$ and IOL power $(0.07 \mathrm{D} \pm 0.26 \mathrm{D})$, followed by the LENSTAR-immersion comparison $(0.04 \mathrm{~mm} \pm 0.17 \mathrm{~mm} ;-0.08 \mathrm{D} \pm 0.75 \mathrm{D})$ and the LENSTAR-applanation comparison $(0.18 \mathrm{~mm} \pm 0.23 \mathrm{~mm}$; $-0.52 \mathrm{D} \pm 0.93 \mathrm{D})$. IOL power values obtained using LENSTAR therefore tend to be smaller than those of ultrasound biometry, particularly applanation. For average $\mathrm{K}$, the LENSTAR-IOLmaster comparison gives slightly larger magnitude of mean difference but much smaller SD $(-0.11 \mathrm{D} \pm 0.18 \mathrm{D})$ compared with the LENSTAR-TOPCON comparison (0.04 D $\pm 0.41 \mathrm{D})$. The distributions of the differences in all these comparison groups are approximately symmetric (see Supplementary Material).

The Bland-Altman plots (Figure 1) show that the width of the $95 \%$ LoA for AL difference is narrowest (about $0.1 \mathrm{~mm}$ ) in the LENSTAR-IOLMaster comparison, and $\sim 0.7 \mathrm{~mm}$ and $0.9 \mathrm{~mm}$ in the LENSTAR-immersion and LENSTAR-applanation comparisons, respectively. Figure 2 shows that the width of the 95\% LoA for IOL power difference is narrowest $(1 \mathrm{D})$ for the LENSTARIOLMaster comparison, followed by LENSTARimmersion (3 D) and LENSTAR-applanation (3.6 D) comparisons. We note an exceptionally large difference (close to $-5 \mathrm{D}$ ) in the LENSTAR-applanation comparison, which belongs to a subject with long AL (about $28 \mathrm{~mm}$ ). The same subject also produced the positive difference outlier in the LENSTAR-applanation $(+1.11 \mathrm{~mm}$ difference) and LENSTAR-immersion comparison $(+0.78 \mathrm{~mm})$ in Figure 1 . Figure 3 shows the Bland-Altman plot for average $\mathrm{K}$ in the LENSTARIOLMaster and LENSTAR-TOPCON comparisons. The width of the $95 \%$ LoA is narrowest in the LENSTARIOLMaster comparison (about 0.7 D; $1.6 \mathrm{D}$ for LENSTAR-TOPCON).

Table 1 shows the proportions of differences falling within three ranges of IOL power in the three comparisons. We note that the LENSTAR-IOLMaster comparison gives the most desirable result, followed by the LENSTAR-immersion and LENSTAR-applanation comparisons. The result of Fisher's exact test for the $3 \times 3$ table is statistically significant $\left(P\right.$-value $\approx 10^{-14}$ ); there is association between the three paired comparison groups with the proportion of difference measures that indicate clinically important levels of disagreement. The negligible $P$-value is expected because there are two adjacent cells with zero counts in the LENSTARIOLMaster comparison. To see if the association remains after removal of the latter, we performed Fisher's exact test on the $2 \times 3$ table without the LENSTAR-IOLMaster comparison; the result is still statistically significant $(P$-value $=0.02)$, thus supporting the ranking implied by casual inspection of the estimated proportions in Table 1.

Table 2 gives the estimated proportion of differences falling within clinically acceptable ranges for the three 

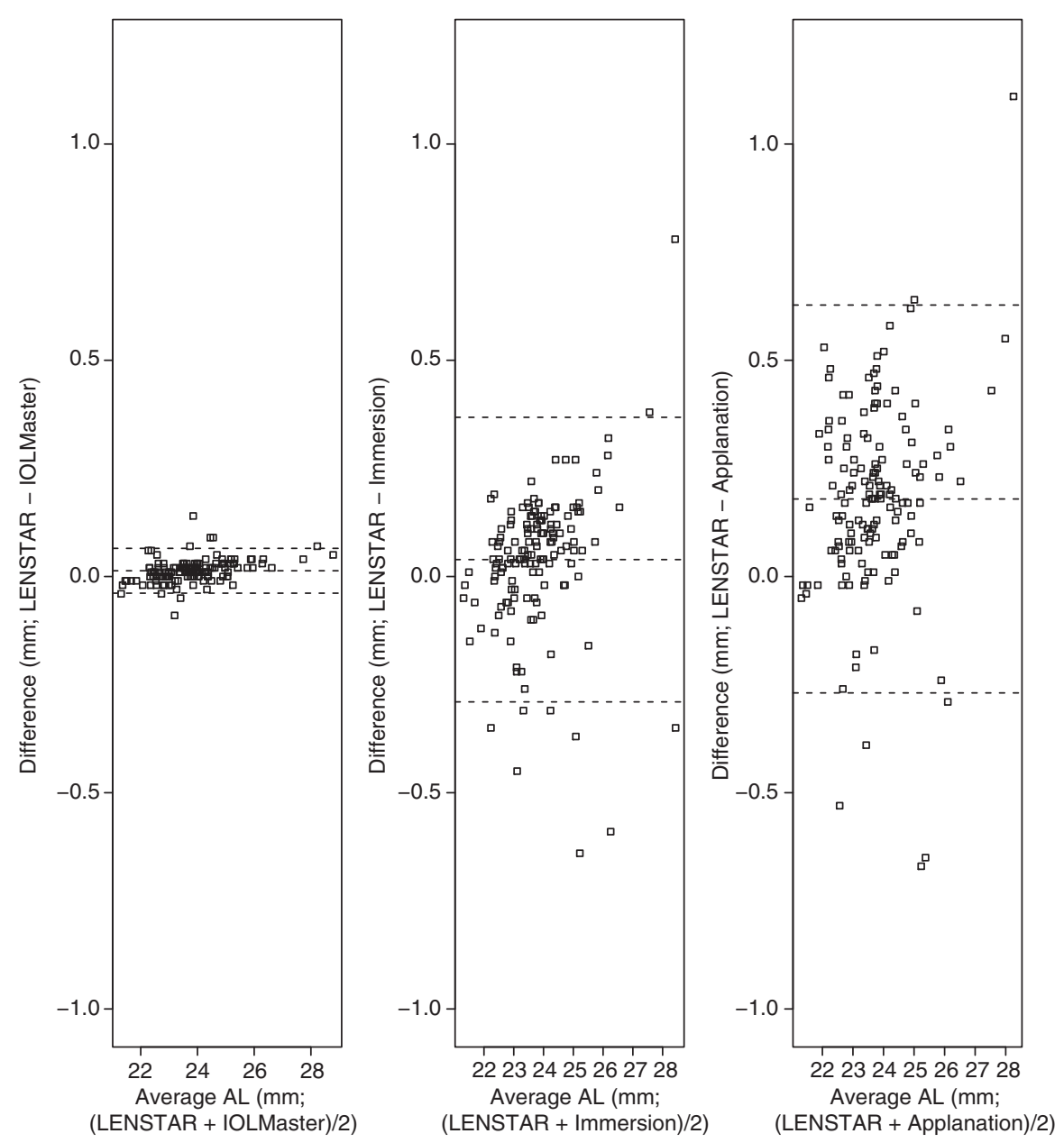

Figure 1 Bland-Altman plots for assessing agreement of LENSTAR-IOLMaster, LENSTAR-applanation, and LENSTAR-immersion comparisons in AL. (95\% LoA for AL difference: LENSTAR-IOLMaster (-0.04, 0.07); LENSTAR-immersion (-0.29, 0.37); LENSTARapplanation $(-0.27,0.63))$

comparisons. The magnitude of discrepancy in the proportion of differences between two comparison groups $\left(\widehat{P_{1}}-\widehat{P_{2}} \pm S E\right)$ informs us about its practical significance. The proportion of AL differences falling within $0.33 \mathrm{~mm}$ from zero in the LENSTAR-IOLMaster comparison is $0.24 \pm 0.04$ more than the LENSTARapplanation comparison, but only $0.05 \pm 0.02$ more than the LENSTAR-immersion comparison. However, for the more stringent cut-off of within $0.1 \mathrm{~mm}$, that of LENSTAR-IOLMaster is $0.48 \pm 0.04$ more than LENSTARimmersion; and $0.74 \pm 0.04$ more than LENSTARapplanation. For the LENSTAR-immersion comparison, the proportion is $0.19 \pm 0.04$ more than LENSTARapplanation comparison for the within $0.3 \mathrm{~mm}$ category; and $0.26 \pm 0.05$ more for the within $0.10 \mathrm{~mm}$ category. For average $\mathrm{K}$, the proportion in the LENSTAR-IOLMaster comparison is only $0.02 \pm 0.02$ more than the LENSTARTOPCON comparison for the within $1 \mathrm{D}$ category-a practically insignificant result. However, it is $0.10 \pm 0.03$ more when we consider the within $0.5 \mathrm{D}$ category. For IOL power difference, the proportion of differences falling within $1 \mathrm{D}$ from zero for the LENSTAR-IOLMaster comparison (Table 1) is $0.32 \pm 0.04$ more than the LENSTAR-applanation comparison, but just $0.18 \pm 0.03$ more than the LENSTAR-immersion comparison; the same proportion is $0.14 \pm 0.05$ more in the latter compared with the LENSTAR-applanation comparison.

\section{Discussion}

In this study, we have presented estimates of a clinically meaningful quantity: the proportion of differences falling within some clinically acceptable range for AL, IOL power, and average $\mathrm{K}$. We suggest that this approach complements the Bland-Altman plots in assessing method agreement under a clinical context, and leads to a more coherent interpretation of the results of method agreement between LENSTAR and other techniques of 

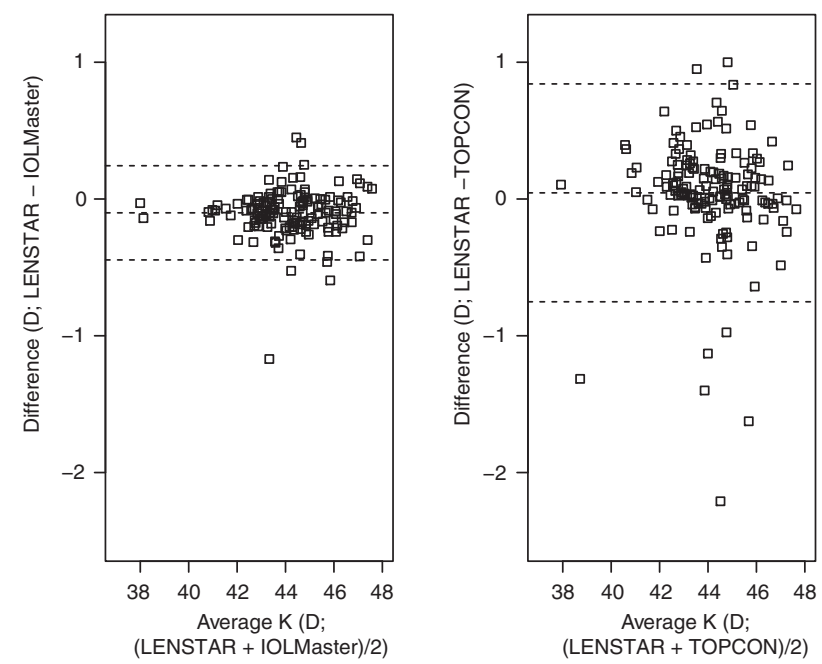

Figure 2 Bland-Altman plots for assessing agreement of LENSTAR-IOLMaster, LENSTAR-applanation, and LENSTARimmersion comparisons in IOL power. When a coordinate has more than one data point, the number of whiskers emanating from a square indicates the number of points stacked together. (95\% LoA for IOL power difference: LENSTAR-IOLMaster $(-0.45,0.59)$; LENSTAR-immersion $(-1.56,1.39)$; LENSTARapplanation $(-2.35,1.31))$

biometry. The mean difference parameter is informative in method agreement studies if two comparisons differ substantially in it; otherwise, it is inadequate. For example, two comparisons can both have zero mean difference, but with substantially different SD. Clearly, agreement between methods in the comparison group with the smaller SD is stronger. By considering the proportion of differences falling within some clinically acceptable range (Tables 1 and 2), we have a simple means of judging method agreement that takes into account both the mean difference and SD, although remaining easy to interpret from the subject matter point of view. As far as we know, no authors have stressed on this point when making agreement analysis between LENSTAR and other biometry techniques.

In judging the merit of our present findings, some aspects of the design of the present study need to be considered. Owing to tight clinic schedules, we did not restrict comparisons using only patients with the same degree of cataract severity; neither did we control for potential effects of age, gender, or ethnicity. As media density in lens with cataract affects the speed of ultrasound, we therefore cannot completely rule out bias in the agreement results involving ultrasound biometry. We also did not randomise the order of biometry. In the context of this problem, however, it seems that applanation must be applied last to avoid the possibility of corneal indentation affecting the outcome of other biometry techniques. Immersion was third in the order because we wanted to limit the potential effect of the immersion liquid on measurement variation between techniques. Hence, randomisation of the order of applying LENSTAR and IOLMaster should have been done. However, we think interpretation of our results is not seriously affected by this shortcoming, as the results of LENSTAR-IOLMaster agreement are similar to those of Holzer et $a l^{10}{ }^{10}$ where randomisation of the order of these two devices was done. The lack of repeatability tests is a weakness too, but does not seem critical as repeatability of LENSTAR has been shown to equal to or better than those of IOLMaster and ultrasound biometry. $^{22}$

Although we did not perform pre and postoperation comparison of IOL power, the prospect of LENSTAR achieving high accuracy in targeted refraction seems high because of its strong agreement with IOLMaster. In a comparative study of 50 cataractous eyes, pre and postoperatively, Packer et $a l^{23}$ found that $92 \%$ of them were within $0.50 \mathrm{D}$ and $100 \%$ were within $1 \mathrm{D}$ of the targeted refraction; IOL power was computed from the IOLMaster using the Holladay II formula. Two other studies $^{7,24}$ showed that a high percentage of patients (87 and 91\%) assigned to IOLMaster biometry achieved targeted refraction of within $1 \mathrm{D}$, compared with about $80 \%$ in the applanation group. In another study, ${ }^{25}$ the two figures were even reported to be as high as 96 and $93 \%$, respectively. For immersion biometry, Haigis et al. ${ }^{26}$ reported that postoperative refraction was correctly predicted within $1 \mathrm{D}$ in $86 \%$ of patients and in $99 \%$ of patients within $2 \mathrm{D}$.

Our results reinforce earlier studies ${ }^{10-14}$ reporting the strong agreement found between LENSTAR and IOLMaster in AL, average $K$, and IOL power. An exception is a recent study, ${ }^{27}$ which reported less satisfactory average $\mathrm{K}$ agreement between IOLMaster and LENSTAR; the mean difference being $0.67 \mathrm{D}$, with $95 \%$ LoA given by $(0.07,1.20)$. The same study also reported similar 95\% LoA in IOL power and AL for their LENSTAR-IOLMaster and LENSTAR-applanation comparison, which differs from the present findings. We believe this discrepancy is caused by the authors' use of two different values of corneal refractive index - same ones in IOLMaster and their Javal-type keratometer, and different ones between LENSTAR and IOLMaster (see Table 6 in their paper). Consequently, the interpretation of their findings is not straightforward. Indeed, Buckhurst et al..$^{22}$ cautioned that using the default setting of corneal refractive index used in LENSTAR (1.332) and IOLMaster (1.3375) can lead to a mean difference of magnitude $0.76 \mathrm{D}$ in average $\mathrm{K}$ in their study population.

Our study suggests that IOL power disagreement between LENSTAR and applanation and immersion ultrasound biometry is a potential source of error 

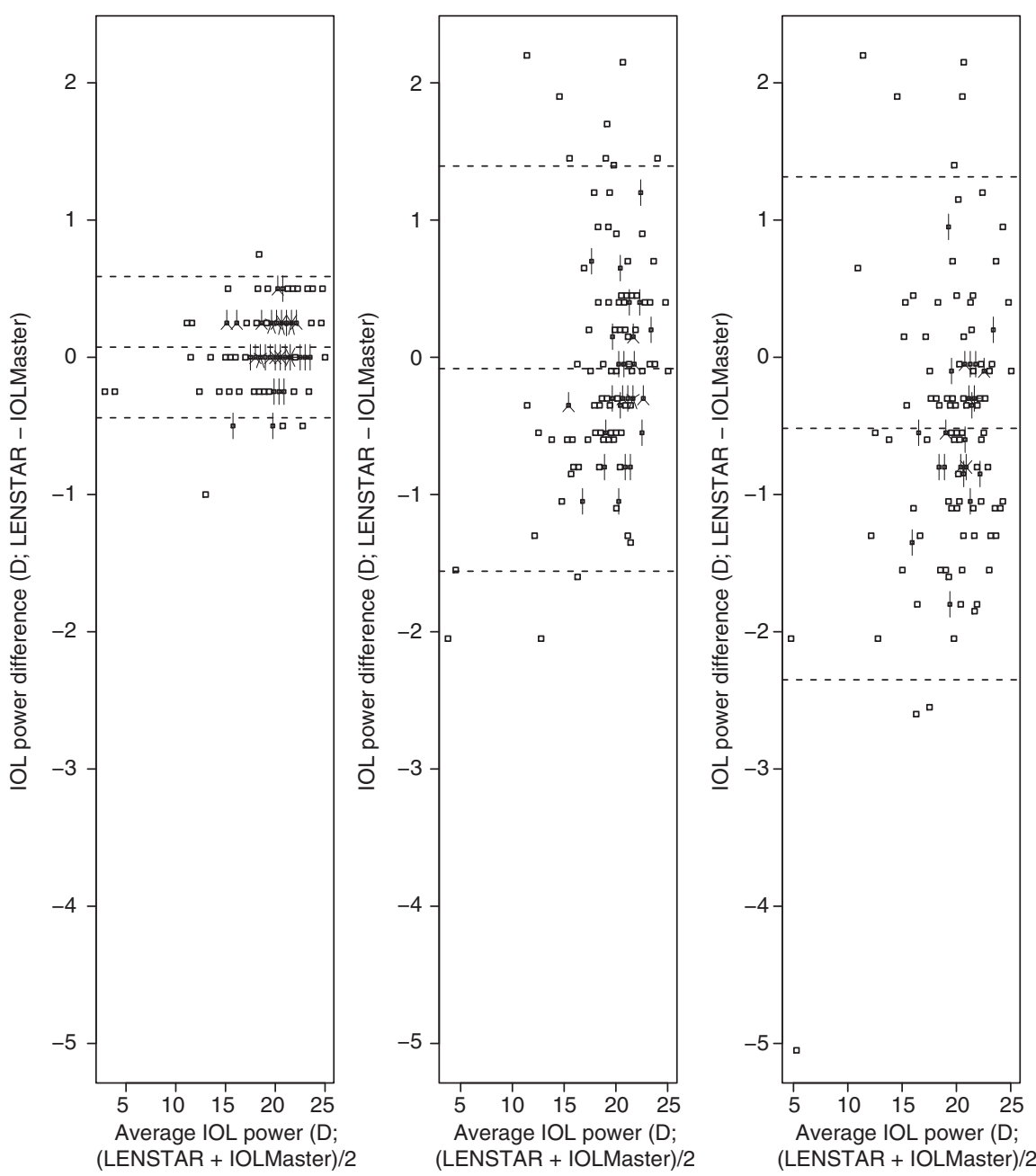

Figure 3 Bland-Altman plots for assessing agreement of LENSTAR-IOLMaster and LENSTAR-TOPCON in average K. (95\% LoA: LENSTAR-IOLMaster $(-0.44,0.24)$; LENSTAR-TOPCON $(-0.75,0.84))$

Table 1 Agreement between methods for IOL power as judged by the estimated proportion of differences falling within clinically acceptable difference ranges from zero $(n=142$ in all comparisons)

\begin{tabular}{lccc}
\hline Comparisons & \multicolumn{2}{c}{$\begin{array}{c}\text { Counts (proportion) of IOL power differences } \\
\text { that fall within specified ranges from zero }\end{array}$} \\
\cline { 2 - 4 } & Within 1 & $\begin{array}{c}\text { Greater than } 1 \mathrm{D} \\
\text { but within } 2 D\end{array}$ & $\begin{array}{c}\text { Greater } \\
\text { than } 2 D\end{array}$ \\
\hline LENSTAR-IOLMaster & $142(1)$ & 0 & 0 \\
LENSTAR-applanation & $97(0.68)$ & $37(0.26)$ & $8(0.06)$ \\
LENSTAR-immersion & $117(0.82)$ & $21(0.15)$ & $4(0.03)$ \\
\hline
\end{tabular}

contributing to incidences of postoperative refractive surprise. Aside from AL error, keratometry reading error is one of the most common causes of preoperative IOL power error resulting in postoperative lens exchange. ${ }^{28}$ As the built-in keratometer in LENSTAR and IOLMaster estimate K1 and K2 differently from those of a manual keratometer, $^{29}$ some discrepancy is expected. In our study, we found that about nine-tenths of average $\mathrm{K}$ differences in the LENSTAR-TOPCON comparison are within $0.5 \mathrm{D}$ from zero (Table 2). Despite the good agreement in AL (95\% within $0.33 \mathrm{~mm}$ ) between LENSTAR and immersion, reduced agreement in average $\mathrm{K}$ can reduce the final agreement in IOL power calculated using the SRK/T formula. On the other hand, proper optimisation of lens constants (Holladay 2, Hoffer Q, SRK/T, and Haigis formulae) has been reported ${ }^{30}$ to 
Table 2 The empirical proportion of differences that fall within clinically acceptable difference ranges for AL $(n=146)$ and average $\mathrm{K}(n=146$ in LENSTAR-IOLMaster; $n=147$ in LENSTAR-TOPCON)

\begin{tabular}{|c|c|c|c|c|c|}
\hline \multirow[t]{2}{*}{ Comparisons } & \multicolumn{2}{|c|}{$\begin{array}{l}\text { Counts (proportion) of AL differences that } \\
\text { fall within specified ranges from zero }\end{array}$} & \multirow[t]{2}{*}{ Comparisons } & \multicolumn{2}{|c|}{$\begin{array}{l}\text { Counts (proportion) of average K differences } \\
\text { that fall within specified ranges from zero }\end{array}$} \\
\hline & Within $0.33 \mathrm{~mm}$ & Within $0.10 \mathrm{~mm}$ & & Within 1D & Within $0.5 D$ \\
\hline LENSTAR-IOLMaster & $146(1)$ & $145(0.99)$ & LENSTAR-IOLMaster & $145(0.99)$ & $143(0.98)$ \\
\hline LENSTAR-applanation & $111(0.76)$ & $37(0.25)$ & LENSTAR-Topcon & $142(0.97)$ & $129(0.88)$ \\
\hline LENSTAR-immersion & $138(0.95)$ & $74(0.51)$ & & & \\
\hline
\end{tabular}

improve the accuracy of IOL power calculation when ocular variables are measured from highly myopic and hyperopic eyes using IOLMaster.

One of the clearest benefits of new generation optical biometry techniques like LENSTAR and IOLMaster is the potential to minimise measurement variation due to operator experience. Kielhorn et al. ${ }^{31}$ showed that both experienced and inexperienced operators of the IOLMaster returned essentially similar measurements for IOL power. In constrast, Goel et al. ${ }^{32}$ reported that AL measurement variation between expert and non-expert operators for ultrasound biometry was 10 times higher compared with IOLMaster. In overcrowded ophthalmology clinics worldwide, we see clear advantages in adopting optical low-coherence reflectometry (OLCR) or partial coherence interferometry (PCI) devices in consultation sessions. Cataract patients would experience shorter waiting time; there is no risk of contamination and no need for anaesthetic eye drops. These improvements will be well received by patients, as hospital visits are often time consuming, and can be costly and inconvenient if unanticipated side effects occur. The strong agreement between LENSTAR and IOLMaster for the ocular variables considered suggests biometrical equivalence of measurements from both. On the other hand, it seems that the speed difference between them has the potential to translate into considerable time savings over large numbers of patients seen ( $60 \mathrm{~min}$ for every 40 patients seen), if IOLMaster is used. The difference arises because LENSTAR computes $\mathrm{AL}$, IOL power, and $\mathrm{K}$ readings simultaneously, hence if outlying measurements are detected in any of those variables, one has to repeat the whole procedure. IOLMaster does not have this problem, as the measurements are scored in a sequential manner. Nevertheless, the ability of LENSTAR to measure additional variables such as central corneal and lens thickness, which is absent in IOLMaster, may be useful to ophthalmologists who need such information.

Taking previous and the present findings into account, we believe the case for supplanting ultrasound biometry with either LENSTAR or IOLMaster for routine use in cataract patient treatment is strong. Indeed, mean absolute error in IOL power prediction has been shown to be small and comparable between LENSTAR and IOLMaster. ${ }^{13}$ Unfortunately, hospitals in developing countries continue to face fiscal hurdles in procuring these advanced instruments. Consequently, there is a danger that eye care standards remain stagnant in these places whereas eye specialists in advanced countries continue to push standards to a higher level by taking full advantage of accuracy and additional features offered by LENSTAR and IOLMaster. It is time that health policy makers consider investing in these powerful instruments to narrow the gulf in eye care standards between developing and industrialised countries.

\section{Conclusions}

$\mathrm{AL}$, average $\mathrm{K}$ measurements and $\mathrm{IOL}$ power calculations (SRK/T formula) taken from the OLCR device LENSTAR are biometrically equivalent to those of the PCI device IOLMaster, in the sense that interchanging measurements of the same variables has clinically negligible effect. However, this cannot be done between LENSTAR and the applanation and immersion ultrasound biometry without incurring substantial disagreements in the proportion of IOL power differences falling within $1 \mathrm{D}$ from zero.

\section{Summary}

\section{What was known before}

- Agreement studies of LENSTAR with IOLMaster, applanation, and immersion have been made.

- However, these suffer from flaws in statistical reasoning, and do not use the same patients to analyze agreement for all three comparisons.

- Minimal interpretation of the analysis result from a clinical perspective.

What this study adds

- All three comparisons done with the same patients, larger sample size of eyes. This provides the appropriate frame for judging the relative strength in agreement without complications arising from a meta-analysis.

- Additional interpretation of the Bland-Altman results in terms of proportion of differences falling within clinically acceptable ranges for the ocular variables studied. 


\section{Conflict of interest}

The authors declare no conflict of interest.

\section{Acknowledgements}

The present study was funded by the University of Malaya Research Grant. Dr Azida Juana Wan Abdul Kadir, MRCOphth, (Department of Ophthalmology, University of Malaya) provided help in grant-related matters. Dr Von Bing Yap, (Department of Statistics and Applied Probability, National University of Singapore) read the first draft of the manuscript and gave helpful suggestions on statistical presentation. We appreciate constructive comments from two anonymous referees and the editor Douglas Anderson, which greatly assisted us in our revision work. Financial support: University of Malaya Research Grant (UMRG).

\section{References}

1 Vogel A, Dick HB, Krummenauer F. Reproducibility of optical biometry using partial coherence interferometry: intraobserver and interobserver reliability. J Cataract Refract Surg 2001; 27: 1961-1968.

2 Binkhorst RD. The accuracy of ultrasonic measurement of the axial length of the eye. Ophthalmic Surg 1981; 12: 363-365.

3 Shammas HJ. Intraocular Lens Power Calculation. Slack Inc: NJ, 2004.

4 Giers U, Epple C. Comparison of A-scan device accuracy. J Cataract Refract Surg 1990; 16: 235-242.

5 Verhulst E, Vrijghem JC. Accuracy of intraocular lens power calculations using the Zeiss IOL Master. A prospective study. Bull Soc Belge Ophthalmol 2001; 281: 61-65.

6 Tehrani M, Krummenauer F, Blom E, Dick HB. Evaluation of the practicality of optical biometry and applanation ultrasound in 253 eyes. J Cataract Refract Surg 2003; 29: 741-746.

7 Rajan MS, Keilhorn I, Bell JA. Partial coherence laser interferometry vs conventional ultrasound biometry in intraocular lens power calculations. Eye 2002; 16: 552-556.

8 Freeman G, Pesudovs K. The impact of cataract severity on measurement acquisition with the IOLMaster. Acta Ophthalmol Scand 2005; 83: 439-442.

9 Altman DG. Practical Statistics For Medical Research. Chapman and Hall/CRC: Boca Raton, 1991.

10 Holzer MP, Mamusa M, Auffarth GU. Accuracy of a new partial coherence interferometry analyser for biometric measurements. Br J Ophthalmol 2009; 93: 807-810.

11 Rohrer K, Frueh BE, Wälti R, Clemetson IA, Tappeiner C, Goldblum D. Comparison and evaluation of ocular biometry using a new noncontact optical low-coherence reflectometer. Ophthalmology 2009; 116: 2087-2092.

12 Rabsilber TM, Jepsen C, Auffarth GU, Holzer MP. Intraocular lens power calculation: clinical comparison of 2 optical biometry devices. J Cataract Refract Surg 2010; 36: 230-234.

13 Cruysberg LPJ, Doors M, Verbakel F, Berendschot TTJM, De Brabander J, Nuijts RMMA. Evaluation of the
Lenstar LS 900 non-contact biometer. Br J Ophthalmol 2010; 94: 106-110.

14 Hoffer KJ, Shammas HJ, Savini G. Comparison of 2 laser instruments for measuring axial length. J Cataract Refrac Surg 2010; 36: 644-648.

15 Bunce C. Correlation, agreement, and Bland-Altman analysis: statistical analysis of method comparison studies. Am J Ophthalmol 2009; 148: 4-6.

16 Patton N, Aslam T. Statistical analysis of agreement in measurement comparison studies. Eye 2004; 19: 363.

17 Altman DG, Bland JM. Measurement in medicine: the analysis of method comparison studies. Statistician 1983; 32: 307-317.

18 Bland JM, Altman DG. Statistical methods for assessing agreement between two methods of clinical measurement. Lancet 1986; 327: 307-310.

19 Bland JM, Altman DG. Measuring agreement in method comparison studies. Statist Methods Med Res 1999; 8: 135-160.

20 Retzlaff JA, Sanders DR, Kraff MC. Development of the SRK/T intraocular lens implant power calculation formula. J Cataract Refract Surg 1990; 16: 333-340.

21 R Development Core Team. R: A language and environment for statistical computing. Version 2101 . R Foundation for Statistical Computing: Vienna, Austria, 2009.

22 Buckhurst PJ, Wolffsohn JS, Shah S, Naroo SA, Davies LN, Berrow EJ. A new optical low coherence reflectometry device for ocular biometry in cataract patients. $\mathrm{Br} \mathrm{J}$ Ophthalmol 2009; 93: 949-953.

23 Packer M, Fine IH, Hoffman RS, Coffman PG, Brown LK. Immersion A-scan compared with partial coherence interferometry: outcomes analysis. J Cataract Refract Surg 2002; 28: 239-242.

24 Rose LT, Moshegov CN. Comparison of the Zeiss IOLMaster and applanation A-scan ultrasound: biometry for intraocular lens calculation. Clin Exp Ophthalmol 2003; 31: 121-124.

25 Eleftheriadis H. IOLMaster biometry: refractive results of 100 consecutive cases. Br J Ophthalmol 2003; 87: 960-963.

26 Haigis W, Lege B, Miller N, Schneider B. Comparison of immersion ultrasound biometry and partial coherence interferometry for intraocular lens calculation according to Haigis. Grafes Arch Clin Exp Ophthalmol 2000; 238: 765-773.

27 Salouti R, Nowroozzadeh MH, Zamani M, Ghoreyshi M, Salouti R. Comparison of the ultrasonographic method with 2 partial coherence interferometry methods for intraocular lens power calculation. Optometry 2010; 82: 140-147.

28 Jin GJC, Crandall AS, Jones JJ. Intraocular lens exchange due to incorrect lens power. Ophthalmology 2007; 114: 417-424.

29 Hill WE. Accurate keratometry readings. Cataract refract surg today, June 2009 32-34, available online at http:/ / www.bmctoday.net/crstoday/2009/06. Accessed on 29 June 2010.

30 Terzi E, Wang L, Kohnen T. Accuracy of modern intraocular lens power calculation formulas in refractive lens exchange for high myopia and high hyperopia. J Cataract Refrac Surg 2009; 35: 1181-1189.

31 Kielhorn I, Rajan MS, Tesha P, Subyran VR, Bell JA. Clinical assessment of the Zeiss IOLMaster. J Cataract Refract Surg 2003; 29: 518-522.

32 Goel S, Chua C, Butcher M, Jones CA, Bagga P, Kotta S. Laser vs ultrasound biometry-a study of intra- and interobserver variability. Eye 2004; 18: 514-518. 